\title{
A structure dynamic model updating method for random vibration fatigue life prediction
}

\author{
Tao $\mathrm{Wu}^{1}$, Weixing $\mathrm{Yao}^{1}$, Ziran $\mathrm{Xu}^{2}$, Chao $\mathrm{Xu}^{3}$, and Jie Huang ${ }^{3}$ \\ ${ }^{1}$ Nanjing University of Aeronautics and Astronautics \\ ${ }^{2}$ Shanghai Electro-Mechanical Engineering Institute \\ ${ }^{3}$ Nanjing University of Aeronautics and Astronautics College of Aerospace Engineering
}

October 27, 2020

\begin{abstract}
In order to overcome the low precision of vibration fatigue life analysis of complex engineering structures, a structural dynamic model updating method for random vibration fatigue life prediction was established. In this model updating method, the stiffness and modal damping of key parts such as structural connection are extracted as model modification parameters. The approximate model of modified parameters and target is established to replace the finite element model of complex structure based on the Kriging method. The actual natural frequency and random vibration response spectrum of the structure are taken as the modified objectives to design the optimization experiment and find the optimal model parameters. And based on the amplitude probability density method of random vibration fatigue life analysis, the vibration fatigue life of complex structure is obtained. Finally, the method is verified by structural dynamic experiment. The result shows that the method can effectively modify the parameters of dynamic finite element model for the prediction of structural vibration fatigue life, and improve the accuracy of structural random vibration fatigue life prediction.
\end{abstract}

\section{A structure dynamic model updating method for random vibration fatigue life prediction}

Tao $\mathrm{Wu}^{1}$, Weixing $\mathrm{Yao}^{2}$, Ziran $\mathrm{Xu}^{3}$, Chao $\mathrm{Xu}^{1}$, Jie Huang ${ }^{2}$

(1 Key Laboratory of Fundamental Science for National Defense-Advanced Design Technology of Flight Vehicle, Nanjing University of Aeronautics and Astronautics, Nanjing 210016, China;

2 State Key Laboratory of Mechanics and Control of Mechanical Structures, Nanjing University of Aeronautics and Astronautics, Nanjing 210016, China;

3 Shanghai Electro-Mechanical Engineering Institute, Shanghai 201109, China)

Abstract: In order to overcome the low precision of vibration fatigue life analysis of complex engineering structures, a structural dynamic model updating method for random vibration fatigue life prediction was established. In this model updating method, the stiffness and modal damping of key parts such as structural connection are extracted as model modification parameters. The approximate model of modified parameters and target is established to replace the finite element model of complex structure based on the Kriging method. The actual natural frequency and random vibration response spectrum of the structure are taken as the modified objectives to design the optimization experiment and find the optimal model parameters. And based on the amplitude probability density method of random vibration fatigue life analysis, the vibration fatigue life of complex structure is obtained. Finally, the method is verified by structural dynamic experiment. The result shows that the method can effectively modify the parameters of dynamic finite element model for the prediction of structural vibration fatigue life, and improve the accuracy of structural random vibration fatigue life prediction. 
Key words :Dynamic model updating; approximate model; vibration fatigue; fatigue life analysis

\section{Introduction}

With the improvement of the design capability of structure, modern engineering structure forms and load environments become more and more complex. In particular, modern aerospace structures often need to bear severe vibration loads, which leads to vibration fatigue failure. Therefore, how to accurately predict the vibration fatigue life of complex structures in the design stage has gradually become a research focus[1-3].

The premise of the accurate vibration fatigue life estimation is the accurate structural dynamic response. The most accurate dynamic response is the measured response, but sometimes the dynamic response of structure can not be measured directly in practical engineering, so the dynamic response must rely on finite element method. Due to the influence of model simplification, manufacturing error, boundary error and other factors, there are very large errors in the process of finite element analysis results, so it is necessary to modify the dynamic finite element model[4,5].

According to the modified object, the current dynamic model updating methods can be divided into matrix method and design parameter method. Matrix method is based on the matrix perturbation theory, and in the method, orthogonality and system characteristic equation are introduced as constraints to modify stiffness matrix and mass matrix [6-9]. Among them, Berman [6] first proposed a theoretical model method for identifying dynamic parameters of linear elastic systems based on measured modal data. However, the mass or stiffness matrix modified by this method changes the banded and sparse properties of the original matrix, and the physical meaning of the modified matrix is not clear, so it is difficult to be applied to engineering practice, and rarely used at present [10-12]. The design parameter method takes the inherent properties of the structure (such as modulus, density, etc.) as the modified parameters, and obtains the optimal design parameters through analytical calculation or finite element iterative calculation [13-18]. Fox [15] analyzed the change rate of the correlation between structural vibration eigenvalues and structural parameters by analytical method, which is also the primary work of dynamic model updating based on design parameters. Fang [18] applied the D-optimal test design method to solve the model updating problem, and verified the effectiveness of the method by using reinforced concrete frames. For the complex modern engineering structures, the analytical method is hardly applicable. At present, the dynamic model updating based on design parameters is mainly modeled by finite element method and combined with optimization theory to find the optimal parameters [19-21]. In the existing research, most of the modified objectives of the model are natural frequencies. For the fatigue life analysis of complex structures under random vibration load, accurate calculation of some natural frequencies can not guarantee the accuracy of structural random response [22-25]. Therefore, it is necessary to update the dynamic model of the structure according to the random vibration response of the structure.

Aiming at improving the accuracy of vibration fatigue life prediction for complex aerospace structures, a dynamic model updating method for random vibration fatigue life analysis is proposed. This method takes the multiple joint stiffness and modal damping as correction parameters. Measured natural frequency and random vibration response spectrum are taken as objective functions. Kriging approximation model is used to replace the complex mapping relationship between parameters and objective functions. And based on Kriging model, the design parameters are optimized by genetic algorithm. Finally, the fatigue life of structure random vibration is predicted by amplitude distribution method and the accuracy of the vibration fatigue life prediction are verified by the dynamic experiment.

\section{Dynamic model updating method}

At present, the dynamic model modification mainly relies on the finite element technology to modify the design parameters directly. Although this method could assure the precision, it would need many iterations 
in the process of model updating. For complex structures, the finite element model is more complex, that is to say, the mapping relationship between design parameters and structural response is complex, and iterative calculation needs considerable time cost. Therefore, an approximate model is derived to replace the complex finite element model. An optimization method is also used to find the optimal parameters. Based on this correction strategy, the dynamic model modification process is established, as shown in Figure 1.

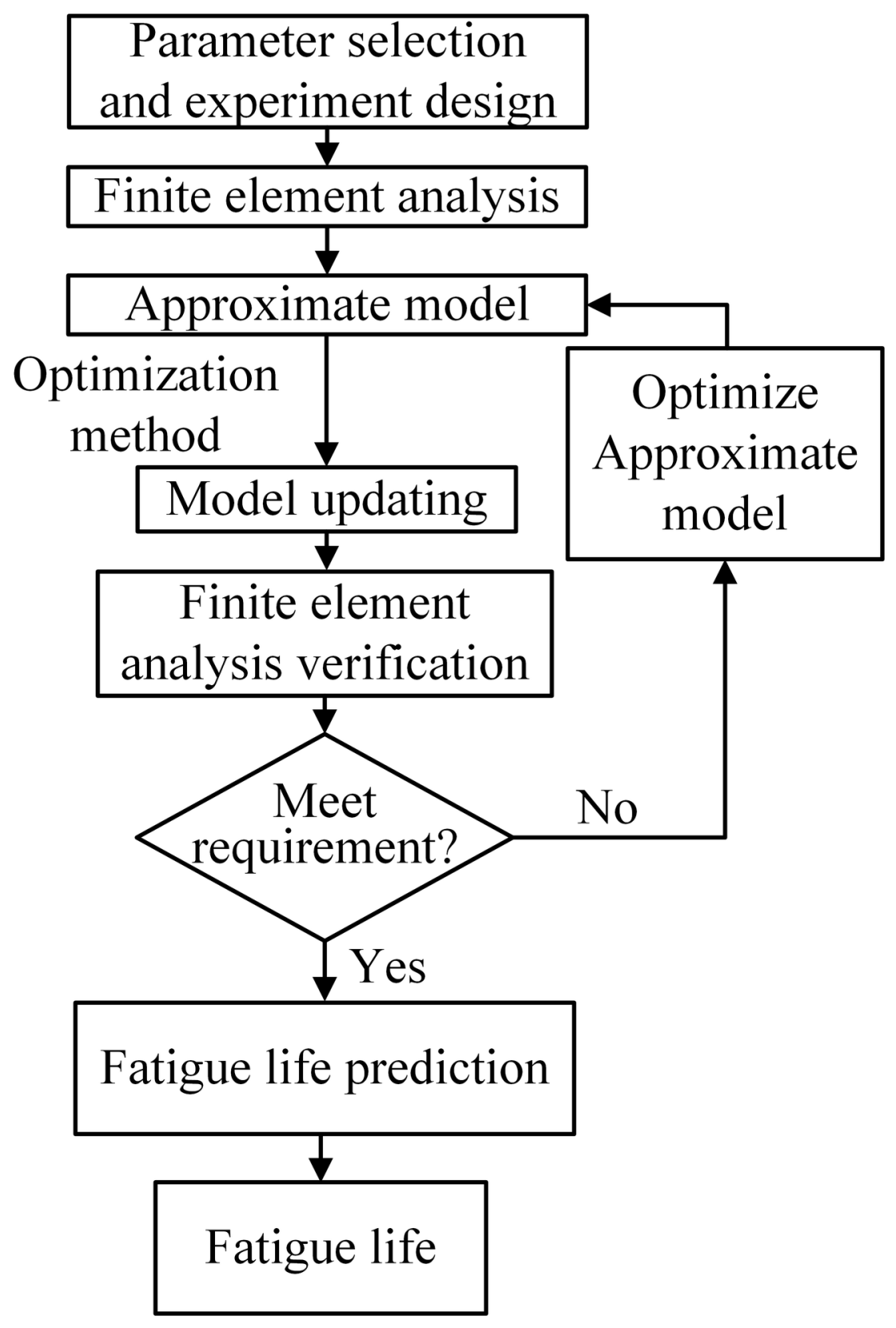

Figure 1 Model updating flow chart based on approximate model and optimization theory

In the updating process, the appropriate design parameters and their ranges are selected for several times of finite element calculation to construct the approximate model. Then the optimal parameters are obtained by optimization method. Finally, the results of finite element and approximate model are compared. If 
the requirements are met, the next step of structural random vibration fatigue life analysis is carried out. Otherwise, the process is repeated after adjusting the approximate model.

The key of the whole model is to construct approximate model and design parameter optimization test (so as to find the optimal parameters). The commonly used approximate modeling techniques include radial basis function method, Kriging method and response surface method (RSM). Kriging model considers that the distribution law of random variables is affected by a certain local deviation in the technology of global expectation. The model also assumes that the local deviation is Gaussian random distribution, and the correlation degree is expressed by covariance. This method is widely used in model updating. In this paper, Kriging approximate model is used to replace the complex mapping relationship between design parameters and structural response.

The purpose of parameter optimization experiment is to find the optimal parameters within the constraint range of design parameter $X_{\mathrm{i}}$, so that the modified model can accurately simulate the actual structural response. In order to evaluate the error between the corrected results and the test data, the natural frequency error coefficient $F_{\text {fi }}$ is defined to solve the problem of structural random vibration fatigue life analysis.

Where $F$ ti is the measured value of the $i$-th natural frequency and $F$ si is the calculated value of the $i$-th natural frequency.

For the problem of random vibration fatigue life prediction, accurate calculation of natural frequency can not guarantee the accuracy of structural response spectrum, while the prediction of random vibration fatigue life needs accurate stress response spectrum of dangerous points. Therefore, the error coefficient $P_{\mathrm{Pi}}$ (Figure 2 ) is defined according to the difference area between the measured acceleration power spectrum and the calculated acceleration power spectrum, which is used to describe the relative error between the calculated and measured power spectral density functions.

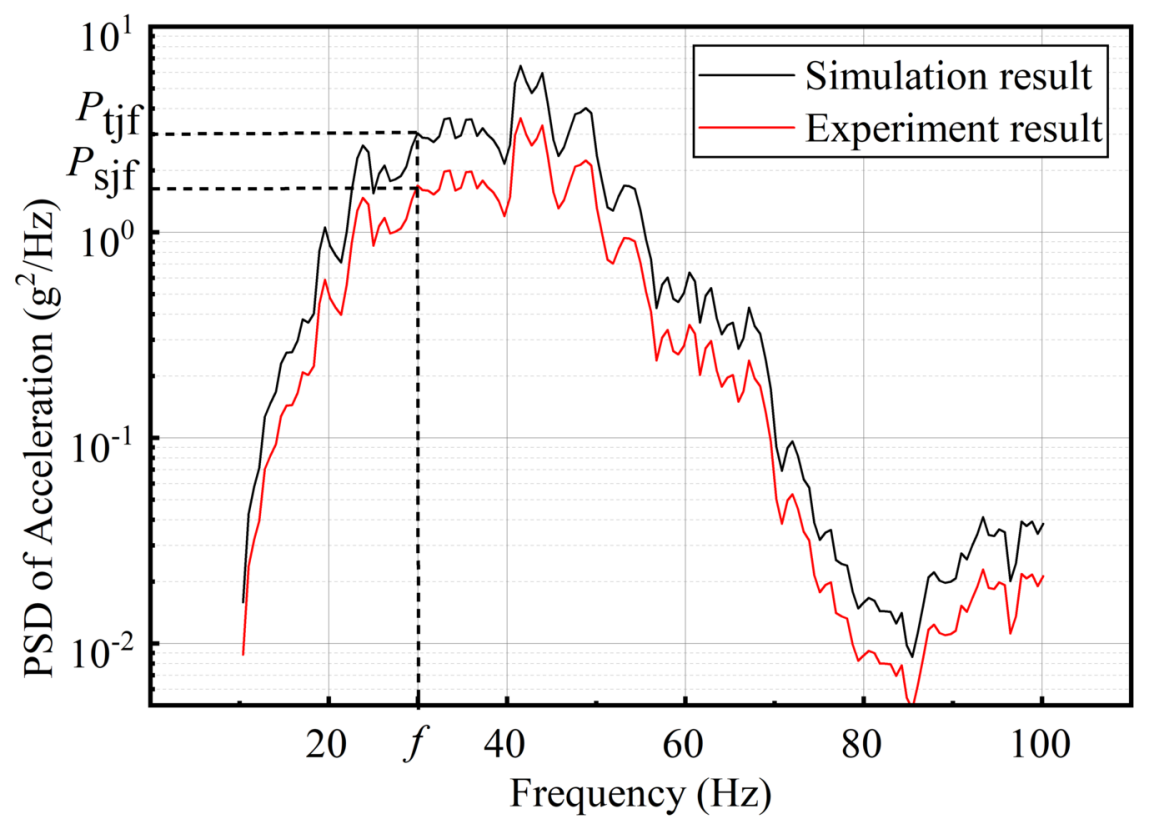

Figure 2 Definition of response spectrum error

The mathematical model of design parameter optimization test can be expressed as

Where $X_{i}$ is the design parameter, $X_{\min }$ and $X_{\max }$ are the upper and lower limits respectively. In the design parameter optimization experiment, genetic algorithm or other optimization methods can be used 
to find the optimal parameters, and then the accurate structural response can be obtained by substituting them into the finite element method, so as to calculate the structural vibration fatigue life.

\section{Structure dynamic test}

\subsection{Test structure and test equipment}

In order to verify the effectiveness of this method, a structural dynamic test was carried out on a complex aerospace structure. The diagram of test structure, acceleration sensor location and fatigue crack diagram are shown in Figure 3. The test object is a thin-walled cylinder structure, which is composed of 5 compartments from left to right. These compartments are connected by stud axial bolts, and the equipment support and equipment are installed inside. During the dynamic test, the whole structure is fixed on the shaking table by four lifting lugs through truss clamp, and then the dynamic test is carried out.

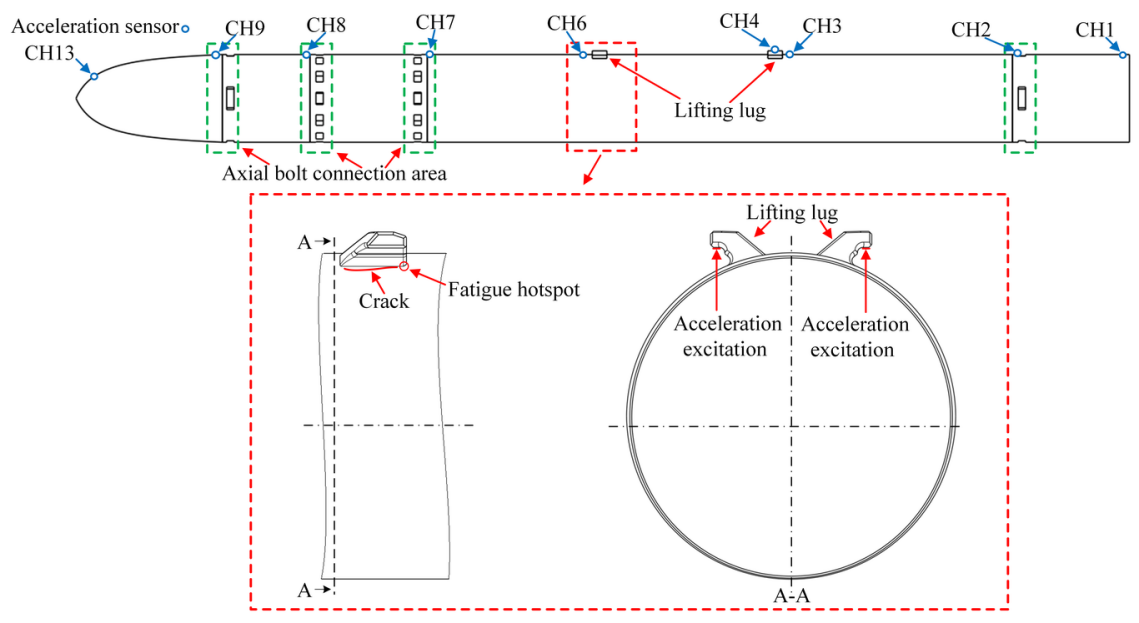

Figure 3 Structure diagram

\subsection{Material properties}

The test object material is $30 \mathrm{Cr} 3 \mathrm{SiNiMoVA}$, and the material properties are shown in Table 1 .

Table 1 Material properties of test structure

\begin{tabular}{ll}
\hline Elastic modulus $E / \mathrm{GPa}$ & 206 \\
\hline Tensile strength $S_{\mathrm{b}} / \mathrm{MPa}$ & 3.14 \\
Poisson ratio $\nu$ & 0.3 \\
Density $\rho / \mathrm{kg} \cdot \mathrm{m}^{-3}$ & 7850 \\
\hline
\end{tabular}

The $S-N$ curve of material $(\mathrm{R}=-1)$ is shown in equation (1). Where $S_{\mathrm{b}}=1715 \mathrm{Mpa}, S_{\mathrm{ae}}=590 \mathrm{MPa}, a=0.4345$, $b=34000$

\subsection{Test process and test results}

The natural frequencies of free modes represent the basic vibration characteristics of the structure. During the test, the natural frequency of free mode of the structure is measured by hammering method, and the first three natural frequencies are shown in Table 2. 
Table 2 Natural frequencies of free modes

\begin{tabular}{ll}
\hline 1st order natural frequency $f_{1}$ & $23.97 \mathrm{~Hz}$ \\
\hline 2nd order natural frequency $f_{2}$ & $58.93 \mathrm{~Hz}$ \\
3rd order natural frequency $f_{3}$ & $102.91 \mathrm{~Hz}$ \\
\hline
\end{tabular}

According to the requirements of GJB150.16, the structure vibration environment test is carried out by loading foundation acceleration excitation on the shaking table. The load control mode is 3,6,13 channel average response control. The acceleration control spectrum and the measured response spectrum in vibration environment test are shown in Figure 4, and the measured response basically reaches the target level of control spectrum.

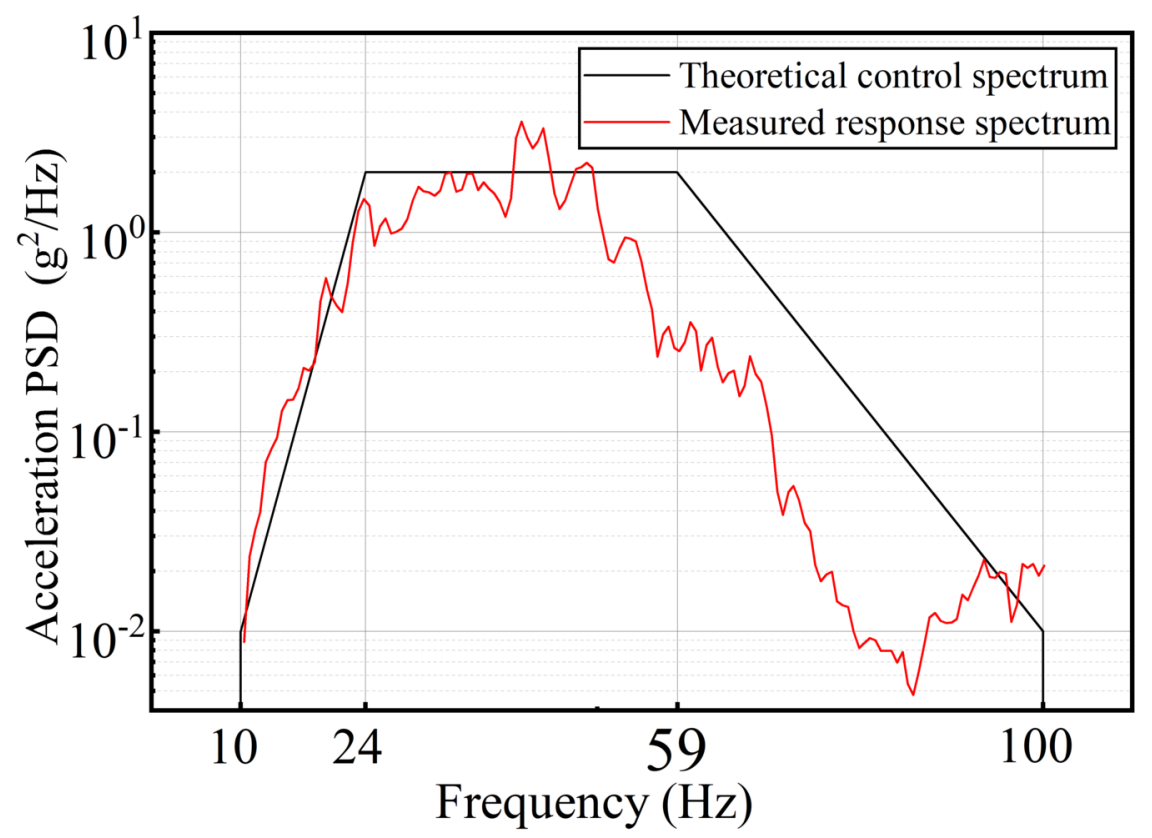

Figure 4 Acceleration control spectrum

After 848 seconds of vibration environment test, obvious cracks appeared at the root of lifting lug, and the structural stiffness decreased significantly, then the test was stopped.

\section{Dynamic model updating and vibration fatigue life analysis}

\subsection{Parameter selection for dynamic model design}

In order to accurately analyze the random vibration fatigue life of lifting lugs, the finite element model of the structure is established to update the dynamic model. The finite element model is shown in Figure 5, and the axial bolts of each connection part are simulated by beam element. 


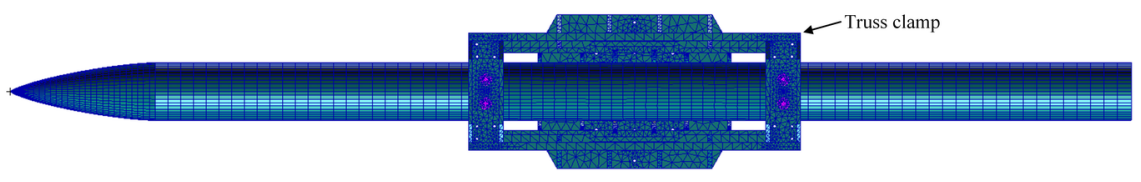

Figure 5 Finite element model

Since there are many equipments in No.4 compartment between $\mathrm{CH} 2$ and $\mathrm{CH} 7$, the integral stiffness is quite different from the finite element model. The other parameters that affect the dynamic characteristics of this structure mainly include the elastic modulus of bolts at each joint, the elastic modulus of test fixture (truss clamp) and the structure modal damping. Therefore, the selection of correction parameters and the range of correction parameters are shown in the Table 3. Since the third natural frequency of the structure has exceeded the maximum frequency range of the load, only the first three modal damping is taken as the correction parameter. In order to establish approximate model, 200 modify parameter test points were selected by the optimal Ladin hypercube sampling method in the experiment design. The optimal Ladin hypercube sampling method ensures that the sample points are full of sample space.

Table 3 Correction parameters and value range

\begin{tabular}{lll}
\hline Modify parameters & Minimum value & Maximum value \\
\hline Elastic modulus of connecting bolt between compartment 1 $\sim 2$ & $100 \mathrm{GPa}$ & $210 \mathrm{GPa}$ \\
Elastic modulus of connecting bolt between compartment 2 $\sim 3$ & $100 \mathrm{GPa}$ & $210 \mathrm{GPa}$ \\
Elastic modulus of connecting bolt between compartment 3 $\sim 4$ & $100 \mathrm{GPa}$ & $210 \mathrm{GPa}$ \\
Elastic modulus of connecting bolt between compartment $4 \sim 5$ & $100 \mathrm{GPa}$ & $210 \mathrm{GPa}$ \\
Elastic modulus of compartment 4 & $210 \mathrm{GPa}$ & $300 \mathrm{GPa}$ \\
Elastic modulus of truss clamp & $100 \mathrm{GPa}$ & $300 \mathrm{GPa}$ \\
1st order modal damping & 0.02 & 0.1 \\
2nd order modal damping & 0.02 & 0.1 \\
3rd order modal damping & 0.02 & 0.1 \\
\hline
\end{tabular}

\subsection{Kriging approximation model}

In order to replace the complex mapping relationship between design parameters and structure response, a Kriging approximate model is established. In the model, first three natural frequencies and the power spectral density function of all sample points are calculated by finite element method, and then Kriging model is generated based on the calculation results. The Kriging results are compared with the finite element results, as shown in Figure 6. According to the comparison, the generated Kriging approximate model can effectively approximate the finite element model within the calculation range. 

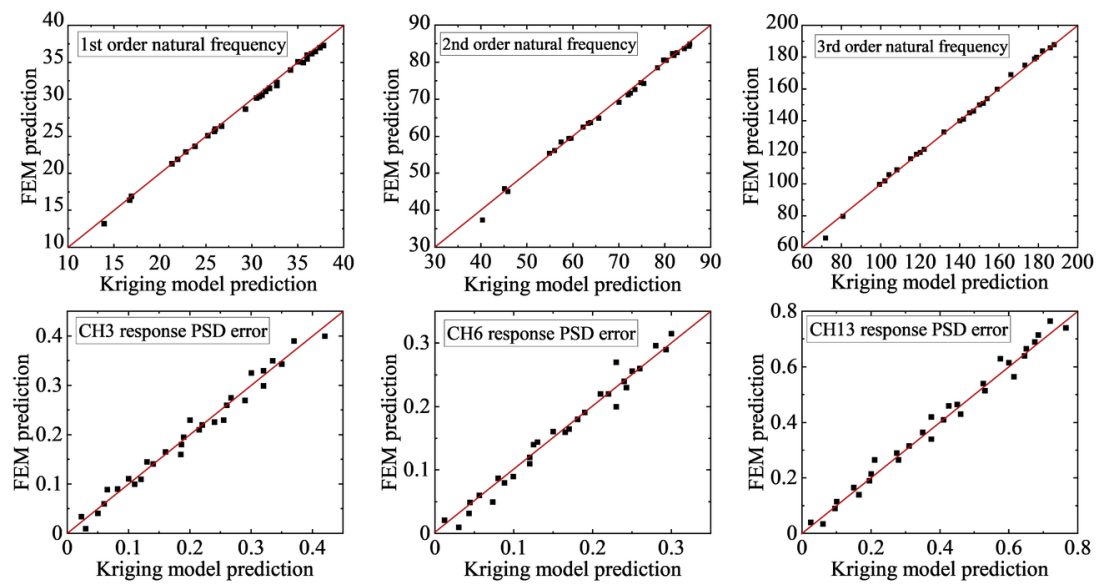

Figure 6 Comparison between Kriging model and finite element method

\subsection{Parameter optimization}

In order to find the optimal parameters, the total error of natural frequency and the response power spectrum are defined as objective functions

The multi island genetic algorithm is used to find the optimal parameters in the generated Kriging model, so that the error objective function has a minimum value. The error convergence curve is shown in Figure

7. The error objective function converges to about 0.57

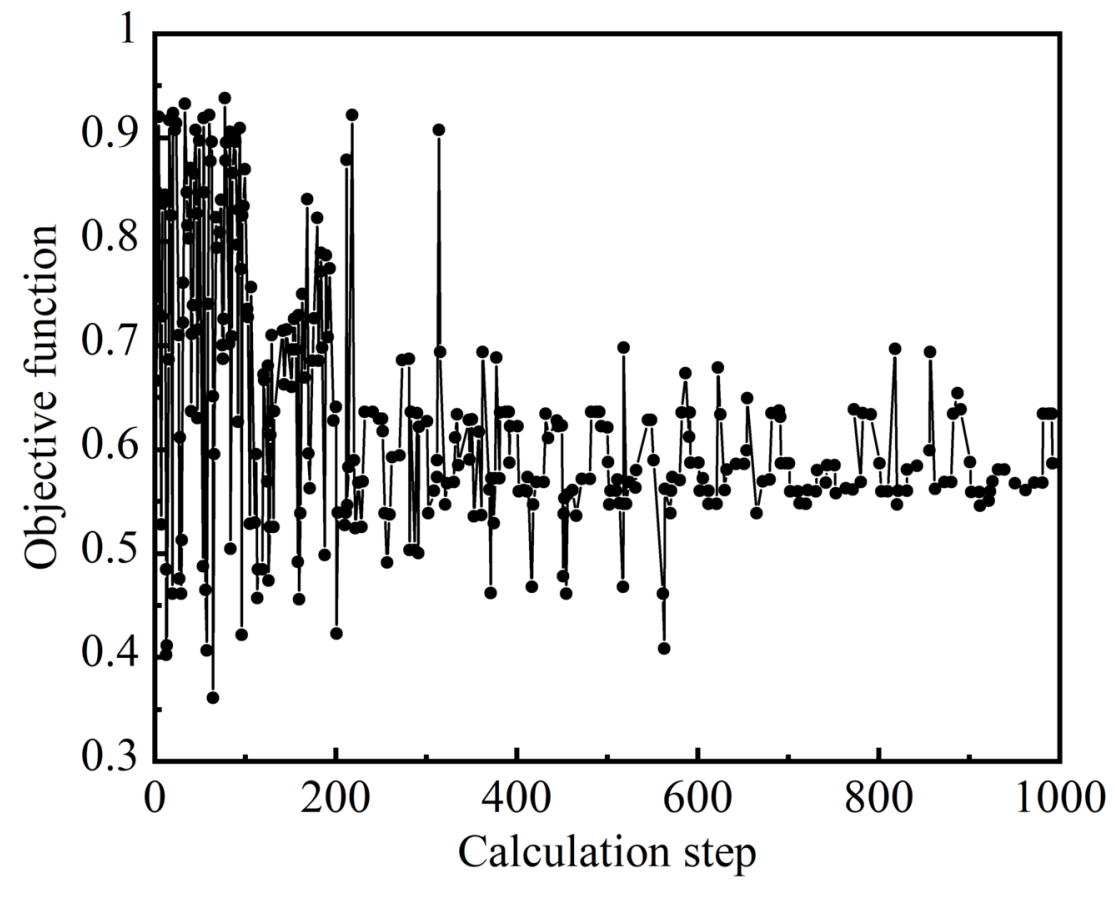

Figure 7 Error objective function calculation

The optimal parameters obtained after correction are as shown in Table 4. 
Table 4 Parameter correction results

\begin{tabular}{lll}
\hline Parameter & Initial value & Correction result \\
\hline Elastic modulus of connecting bolt between compartment 1 $\sim 2$ & $210 \mathrm{GPa}$ & $115 \mathrm{GPa}$ \\
Elastic modulus of connecting bolt between compartment 2 $\sim 3$ & $210 \mathrm{GPa}$ & $105 \mathrm{GPa}$ \\
Elastic modulus of connecting bolt between compartment 3 $\sim 4$ & $210 \mathrm{GPa}$ & $130 \mathrm{Gpa}$ \\
Elastic modulus of connecting bolt between compartment $4 \sim 5$ & $210 \mathrm{GPa}$ & $121 \mathrm{GPa}$ \\
Elastic modulus of compartment 4 & $210 \mathrm{GPa}$ & $265 \mathrm{GPa}$ \\
Elastic modulus of truss clamp & $210 \mathrm{GPa}$ & $236 \mathrm{GPa}$ \\
1st order modal damping & 0.02 & 0.067 \\
2nd order modal damping & 0.02 & 0.075 \\
3rd order modal damping & 0.02 & 0.081 \\
\hline
\end{tabular}

The corrected parameters are substituted into the finite element model to calculate the natural frequency and response power spectrum error, as shown in the Table 5. The calculated control spectrum is shown in Figure 8. It can be seen that the error of finite element calculation response spectrum after correction is greatly reduced compared with that before correction.

Table 5 Updated natural frequency and response spectrum error

\begin{tabular}{llll}
\hline Variable & Measured value & Before updating & After updating \\
\hline 1st order natural frequency & $23.97 \mathrm{~Hz}$ & $28.23 \mathrm{~Hz}$ & $23.97 \mathrm{~Hz}$ \\
2nd order natural frequency & $58.93 \mathrm{~Hz}$ & $75.45 \mathrm{~Hz}$ & $58.91 \mathrm{~Hz}$ \\
1st order natural frequency & $102.91 \mathrm{~Hz}$ & $141.62 \mathrm{~Hz}$ & $106.11 \mathrm{~Hz}$ \\
Response spectrum error & - & 2.89 & 0.57 \\
\hline
\end{tabular}

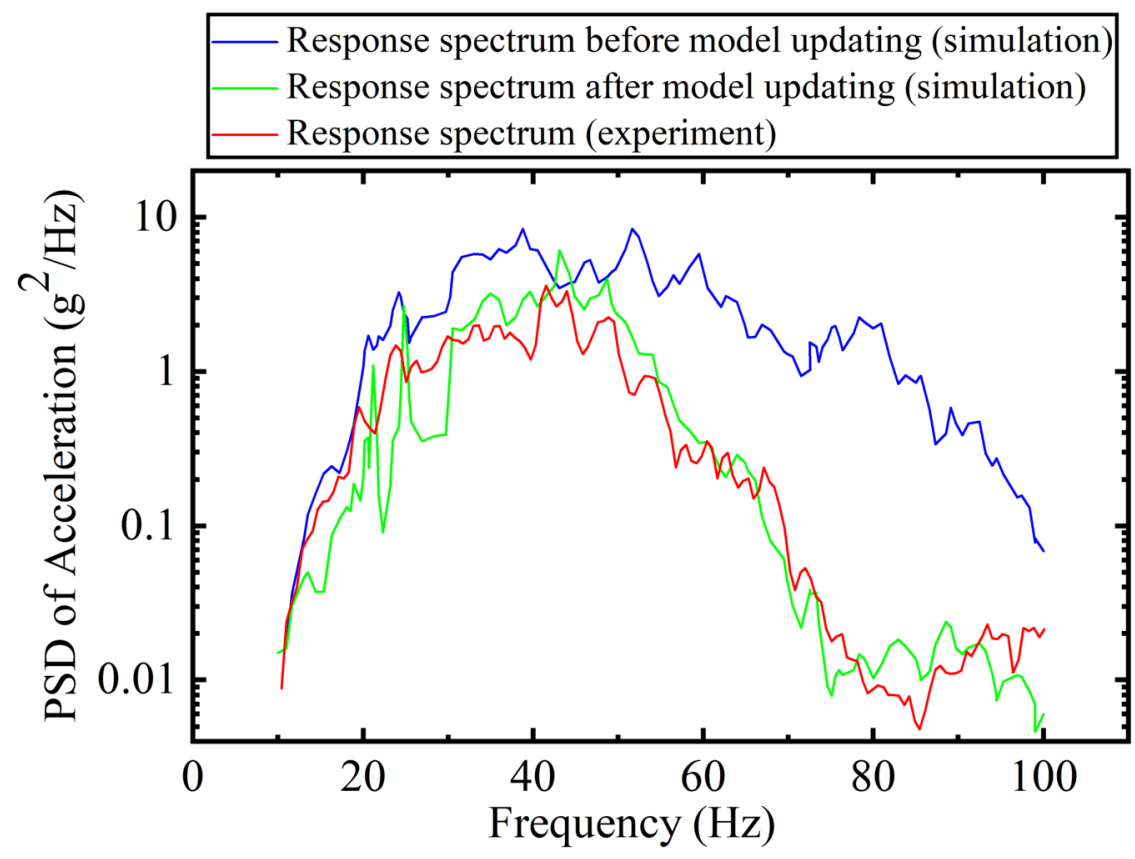

Figure 8 Comparison of finite element calculation of response spectrum before and after correction 


\subsection{Random vibration fatigue life analysis}

Time domain method and frequency domain method are the main methods to estimate the fatigue life of structures under random vibration. Because the time-domain method needs to count a large number of stress-strain history, the frequency-domain method based on stress-strain power spectral density function is widely used. The power spectral density function of stress describes the statistical information of the stress history in the process of random vibration. The probability density function $P(S)$ of the stress in the process of random vibration can be obtained through the distribution model of the amplitude or peak value of the stress, so as to estimate the vibration fatigue life of the structure. Among the probability density distribution models of stress, Dirlik[1] model is the most widely used. There often exists notchs in practical engineering structure. In order to calculate the vibration fatigue life of notched parts, Li Deyong[26] proposed a nominal stress method based on dynamic fatigue notch coefficient, and the life calculation is shown in equation

Where $T$ is the vibration fatigue life $; K$ fis the dynamic fatigue notch coefficient; $P(S)$ is the probability density function of stress amplitude; $D_{1}, D_{2}, D{ }_{3}, \mathrm{Z}, \mathrm{Q}, \mathrm{R}, \gamma$, are the model coefficients obtained from the stress spectral distance. In this paper, the method is used to calculate the random vibration fatigue life of structures.

The result shows that the dynamic stress concentration factor $K$ RMS $=3.36$ and the dynamic fatigue notch factor $K_{\mathrm{f}}=3.33$. The nominal stress response power spectrum of fatigue hotspot before and after correction is shown in Figure 9.

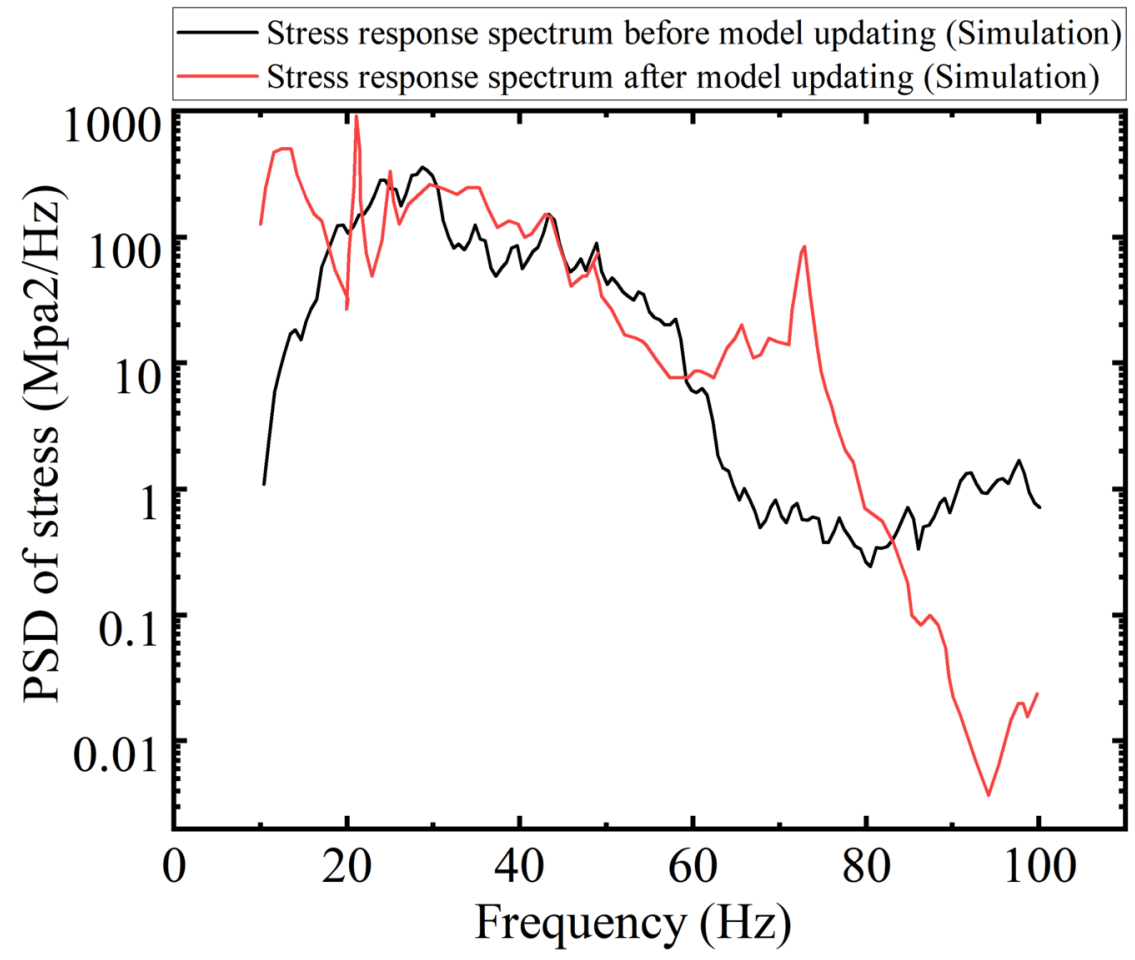

Figure 9 Nominal stress response spectrum of fatigue hotspot before and after model updating

The distribution of stress response amplitude is obtained by Dirlik model, as shown in Figure 10. 


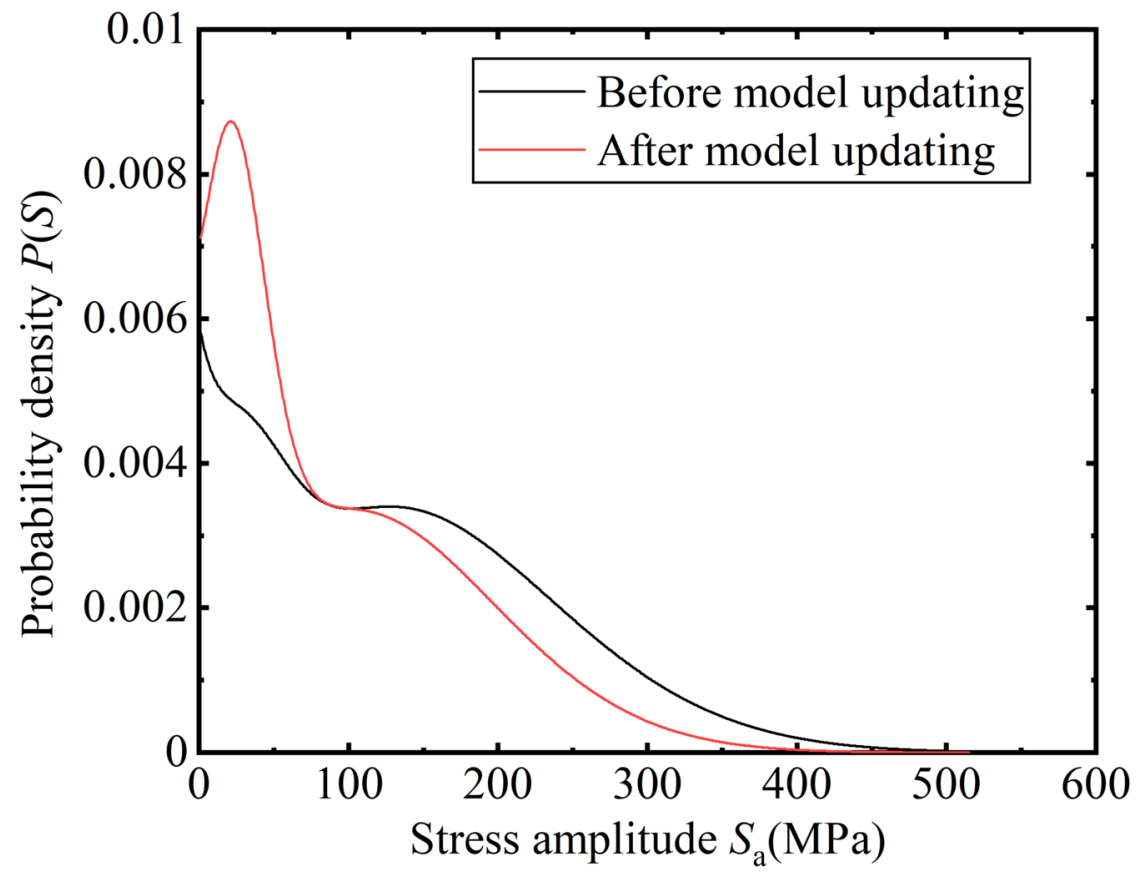

Figure 10 Probability density function of stress amplitude

The calculated vibration fatigue life before and after model modification are obtained by equation, and the calculation results are shown in Table 6 . It can be seen that the calculation accuracy of vibration fatigue life after model modification is greatly improved.

Table 6 Fatigue life prediction and experiment result

\begin{tabular}{lll}
\hline Predicted life before model updating & Predicted life after model updating & Experiment fatigue life \\
\hline $132 \mathrm{~s}$ & $1226 \mathrm{~s}$ & $848 \mathrm{~s}$ \\
\hline
\end{tabular}

\section{Conclusion}

In order to predict structural vibration fatigue life accurately, the dynamic finite element model is updated based on Kriging approximate model and genetic algorithm. Then the vibration fatigue life is calculated based on the updated dynamic finite element model and compared with the dynamic test result. The following conclusions can be drawn:

(1) According to the structural characteristics, the connection stiffness and modal damping factors are selected as the design parameters to establish the Kriging approximate model. The Kriging approximate model can effectively simulate the complex mapping relationship between the structural parameters of the rocket and the modal frequency and the random vibration response error. The Kriging approximate model can be used to modify the dynamic model of the rocket structure.

(2) Taking the structural dynamic response spectrum as the target, the genetic algorithm can be used to update the dynamic model parameters quickly and effectively based on Kriging approximation model.

(3) The dynamic model updating method for random vibration fatigue life prediction can effectively improve the accuracy of vibration fatigue life calculation of complex structure. 


\section{Acknowledgment}

This research was supported by the National Natural Science Foundation of China (52002181).

\section{References:}

[1]. Dirlik, T., Application of computers in fatigue analysis. University of Warwick, 1985.

[2]. Gao, D.Y., Yao, W.X., and Wu, T., Failure analysis on the axial-connected bolts of the thin-walled cylinder under random vibration loading. Engineering Failure Analysis 2019. 105: p. 756-765.

[3]. Gao, D.Y., Yao, W.X., and Wu, T., A damage model based on the critical plane to estimate fatigue life under multi-axial random loading. International Journal of Fatigue 2019.129.

[4]. Mottershead, J.E. and Friswell M.I., Model Updating In Structural Dynamics: A Survey. Journal of Sound \& Vibration, 1993. 167(2): p. 347-375.

[5]. Sinha, J.K. and Friswell, M.I., The use of model updating for reliable finite element modelling and fault diagnosis of structural components used in nuclear plants. Nuclear Engineering \& Design, 2003. 223(1): p. 11-23.

[6]. Berman, A. and Flannelly W.G., Theory of Incomplete Models of Dynamic Structures. Aiaa Journal, 1971.9(8):1481-1487.

[7]. Kabe, A.M. Constrained Stiffness Matrix Adjustment Using Mode Data. AIAA Journal, 1985, 23(9):1431-1436.

[8]. Stetson, K.A. and Palma G.E., Inversion of first-order perturbation theory and its application to structural design. Aiaa Journal, 2012.14(4):454-460.

[9]. Chen, J.C. and Wada B.K., Criteria for Analysis-Test Correlation of Structural Dynamic Systems. Journal of Applied Mechanics Trans of the Asme, 1975. 42(2): p. 101-101.

[10]. Roy, N.A., A Survey of Finite Element Model Updating Methods. 1990. Environmental Testing for Space Programmes Test: Facilities and Methods.

[11]. Yin, H., et al., Model Updating Method Based on Kriging Model for Structural Dynamics. Shock \& Vibration, 2019. 2019: p. 1-12.

[12]. Abdullah, N.A.Z., et al., A review on model updating in structural dynamics. 2015 IOP Conf. Ser.: Mater. Sci. Eng. 100012015.

[13]. Lim, K.B., J.L. Junkins and Wang B.P., Re-examination of eigenvector derivatives. Journal of Guidance Control \& Dynamics, 1987. 10(6): p. 581-587.

[14]. Ojalvo, U. I., Efficient computation of mode-shape derivatives for large dynamic systems. Aiaa Journal, 1987. 25(10): p. 1386-1390.

[15]. Fox, R.L. and Kapoor, M.P., Rates of change of eigenvalues and eigenvectors. Aiaa Journal, 1969. 6(12): p. 2426-2429.

[16]. Nelson, R.B., Simplified calculation of eigenvector derivatives. Aiaa Journal, 2015. 24(9): p. 823-832.

[17]. Leng, J.C., Tian, H.X., Xu, S., Zhou, G.Q., and Zhao, H.F., Dynamics model updating of an offshore platform structure based on optimized Kriging model. Journal of Vibration and Shock, 2019. 38(18): $\mathrm{p}: 18-23$.

[18]. Fang, S. and Perera, R., Damage identification by response surface based model updating using Doptimal design. Mechanical Systems and Signal Processing, 2011. 25(2): p. 717-733. 
[19]. Yuan, Z.X. and Yu, K.P., Finite element model updating of damped structures using vibration test data under base excitation. Journal of Sound \& Vibration, 2015. 340: p. 303-316.

[20]. Astroza, R., Nguyen, L.T. and Nestorović, T., Finite element model updating using simulated annealing hybridized with unscented Kalman filter. Computers \& structures, 2016. 177: p. 176-191.

[21]. Friswell, I. M., Finite Element Model Updating in Structural Dynamics. 1995: Kluwer Academic Publishers.

[22]. Seok, et al., Dynamic analysis of a hollow cylinder subject to a dual traveling force imposed on its inner surface. Journal of Sound \& Vibration, 2015.

[23]. Yao, D.S. and Zhao, L.B., Scheme of Model Updating and Implement for Structural Dynamics Analysis. Applied Mechanics \& Materials, 2013. 252: p. 140-143.

[24]. Smith, M.J. and Hutton, S.G., Frequency modification using Newton's method and inverse iteration eigenvector updating. Aiaa Journal, 2012. 30(7): p. 1886-1891.

[25]. Husain, N.A., Khodaparast, H.H. , and Ouyang, H., Parameter selection and stochastic model updating using perturbation methods with parameter weighting matrix assignment. Mechanical Systems \& Signal Processing, 2012. 32(none): p. 135-152.

[26]. Li, D.Y., and Wang, M.Z., Comparison of random vibration fatigue calculation methods. Jiangsu aviation, 2010(S2): p.78-80. 